\title{
ON THE LIMIT OF A SEQUENCE OF POINT SETS
}

\author{
BY HSÜ PAO-LU
}

A variable point $P_{n}$ is said to approach the point $P$ as its li mit if to an arbitrary positive $\epsilon$ there corresponds an $m$ such that

$$
\overline{P_{n} P}<\epsilon, \quad(n>m) .
$$

In other words, $P$ is to have the property that every neighborhood of it contains almost all* the points $P_{n}$.

In attempting to generalize this definition to a sequence of point sets $M_{1}, M_{2}, \cdots$, one is naturally led to begin with a definition of the neighborhood of a set and then write down (Definition $\mathrm{A}_{0}$ ) the last sentence of the last paragraph, replacing the letter $P$ by $M$.

Definition. By the $\epsilon$-neighborhood of a set $M$ is meant the set of all points which have a distance < $<$ from some point of $M$. We shall denote it by $(\epsilon)_{M}$.

Definition $\mathrm{A}_{0} . A$ point set $M$ is called a limit of the sequence of sets $M_{1}, M_{2}, \cdots$, if every neighborhood of it contains almost all the sets $M_{i}$ as partial sets.

But the above definition is far from being useful, because the limit would then not be unique. In the first place, if the set $M$ is a limit in the sense of Definition $\mathrm{A}_{0}$, and if $M$ has a cluster point $C$, then the set $M-C$ has also the property of being a limit of the sequence. Secondly every set containing $M$ as a partial set is a fortiori a limit.

The first difficulty is overcome by requiring $M$ to be closed, and the second difficulty is met by adding still another condition $(\gamma)$ :

Definition A. $A$ set $M$ is said to be the limit of the sequence of sets $M_{1}, M_{2}, \cdots$, if it has the following properties:

( $\alpha$ ) $M$ is closed.

* Thereby is meant that at most a finite number of the points $P_{\boldsymbol{i}}$ can lie outside the neighborhood. 
( $\beta$ ) For an arbitrary $\epsilon>0,(\epsilon)_{M} \supset M_{i}^{*}$ for almost all indices $i$.

$(\gamma)$ For an arbitrary $\epsilon>0,(\epsilon)_{M_{i}} \supset M$ for almost all indices $i$.

Observe that in the case of a sequence of points, $(\alpha)$ is fulfilled, $(\beta)$ and $(\gamma)$ become equivalent, and the definition reduces to the old one.

The following are immediate consequences of the definition:

(1) If a sequence of sets has a limit, the limit is unique.

(2) If a sequence $\mathfrak{S}$ of point sets has the limit $M$, every partial sequence of $\mathfrak{S}$ has the same limit $M$.

Further results hereby obtained consist of two fundamental criteria for the existence of a limit, when we restrict the sets of the sequence to lying in the same finite region of space. Given a sequence $\subseteq$ of sets $M_{1}, M_{2}, \cdots$, an $L$-point of $\subseteq$ shall be defined as a point which is the limit of a sequence of points $P_{1}, P_{2}, \cdots$, where each $P_{i}$ belongs to the set $M_{i}$.

Theorem A. Let

ऽ:

$$
M_{1}, M_{2}, \cdots
$$

be a sequence of point sets such that all the $M_{i}$ 's lie in the same finite region of space. Then $\mathbb{S}$ has a limit when and only when, whatever partial sequence $\mathfrak{S}_{1}$ be selected from $\mathfrak{S}_{\text {, the set of } L \text {-points }}$ of $\Im_{1}$ coincides with the set of $L$-points of $\subseteq$. The limit of $M_{i}$ is then the set of L-points of $\subseteq$.

TheOREM B. A necessary and sufficient condition for the sequence of sets $M_{i}$, lying in the same finite region of space, to have a limit is that, to an arbitrary positive $\epsilon$, there corresponds an $M_{m}$ such that

$\left(\beta^{\prime}\right)(\epsilon)_{M_{m}} \supset M_{i}$ for almost all indices $i$,

$\left(\gamma^{\prime}\right)(\epsilon)_{M_{i}} \supset M_{m}$ for almost all indices $i$.

Example 1. If each $M_{i}$ is closed, $M_{1}$ is bounded, and $M_{i} \supset M_{i+1}$ for all $i$ 's, then a limit $M$ exists and is equal to the set of points common to all the $M_{i}$ 's.

Example 2. If $M_{i+1} \supset M_{i}$ and all the $M_{i}$ 's lie in the same finite region of space, then a limit $M$ exists and is equal to the closed cover $\dagger$ of the set of points which belong to one of the $M_{i}$ 's.

* Read: "the $\epsilon$-neighborhood of $M$ contains $M_{i}$ as a partial set."

$\dagger$ The closed cover of a set is the sum of the set and its first derived set. 
In the case where each $M_{i}$ is a point, the meaning of Theorem $A$ is obvious, while Theorem B leads directly to the fundamental criterion for the variable point $P_{n}$ to approach a limit, namely, to an arbitrary $\epsilon>0$ there corresponds an $m$ such that $\bar{P}_{n} P_{n^{\prime}}<\epsilon$, provided that $n, n^{\prime}<m$.

But what do these theorems tell us when the set $M_{i}$ corresponds to a point function?

To be exact, consider a sequence of functions $f_{1}(P), f_{2}(P), \cdots$, defined in the same bounded set $N$ of $(n-1)$-dimensional space, and converging toward a limiting function $f(P)$, in each point $P$ of $N$. Moreover, let the functions $f_{i}(P), f(P)$ be bounded; that is, $\left|f_{i}(P)\right|<G,|f(P)|<G$, where $G$ is the same number for all the functions. To each $f_{i}$ corresponds then a bounded set $M_{i}$, formed of the points $\left(x_{1}, x_{2}, \cdots, x_{n-1}, x_{n}\right)$, where $P:\left(x_{1}, x_{2}\right.$, $\left.\cdots, x_{n-1}\right)$ is a point of $N$ and $x_{n}=f_{i}(P)$. Furthermore, all the sets $M_{i}$ lie in the same finite region of space. Let $M$ be the set corresponding to $f$ as $M_{i}$ to $f_{i}$. The following result is immediate. If $f_{i}$ is uniformly convergent, $M_{i}$ has the closed cover of $M$ as its limit. But the converse is not true. For example, let $N$ be the interval $(0,1)$, and

$$
f_{i}=\left\{\begin{array}{c}
\epsilon_{i} \quad \text { when } 0 \leqq x \leqq \eta_{i}, \\
1-\epsilon_{i} \text { when } \eta_{i}<x \leqq 1,
\end{array}\right.
$$

where $\epsilon_{i}>0, \eta_{i}>0, \epsilon_{i}>\epsilon_{i+1}, \eta_{i}<\eta_{i+1}, \lim _{n=\infty} \epsilon_{n}=0, \lim _{n=\infty} \eta_{n}=1 / 2$. Here $f_{i}$ converges non-uniformly, while $M_{i}$ has the closed cover of $M$ as its limit.

Nevertheless it is true that if $N$ is closed and $f$ is continuous, then $f_{i}$ converges uniformly when $M_{i}$ approaches $M$ as its limit. Thus under appropriate restrictions Theorem $B$ is equivalent to the condition for uniform convergence, namely, $f_{i}$ converges uniformly when and only when, to an arbitrary $\epsilon>0$, there corresponds an $m$, independent of $P$, such that

$$
\left|f_{n}(P)-f_{n^{\prime}}(P)\right|<\epsilon, \quad\left(m<n, n^{\prime}\right) .
$$

Under the same restrictions Theorem A may be translated as follows. The function $f_{i}$ is uniformly convergent when and only when, for every sequence of points $P_{1}, P_{2}, \ldots$ of $N$ with the limit $P$, the sequence of numbers $f_{1}\left(P_{1}\right), f_{2}\left(P_{2}\right), \cdots$ has the limit $f(P)$.

National University of Peking, Peiping, China 